OPEN ACCESS

Edited by:

Marilyn Huang,

University of Miami Health System,

United States

Reviewed by:

Komsun Suwannarurk,

Thammasat University, Thailand

Clarissa Polen-De,

Mayo Clinic, United States

*Correspondence:

Jianliu Wang

wangjianliu@pkuph.edu.cn

Jingyi Zhou

sy_zhoujingyi@hsc.pku.edu.cn

${ }^{\text {t}}$ These authors have contributed equally to this work

Specialty section: This article was submitted to Gynecological Oncology, a section of the journal

Frontiers in Oncology

Received: 05 August 2021 Accepted: 17 September 2021

Published: 06 October 2021

Citation:

Xu X, Li X, Zhou J and Wang J (2021) Mechanical Stimulus-Related Risk

Signature Plays a Key Role

in the Prognostic Nomogram

For Endometrial Cancer.

Front. Oncol. 11:753910.

doi: 10.3389/fonc.2021.753910

\section{Mechanical Stimulus-Related Risk Signature Plays a Key Role in the Prognostic Nomogram For Endometrial Cancer}

\author{
Xin $\mathrm{Xu}^{1 \dagger}$, Xingchen $\mathrm{Li}^{1 \dagger}$, Jingyi Zhou ${ }^{1 *}$ and Jianliu Wang ${ }^{1,2^{*}}$ \\ ${ }^{1}$ Department of Obstetrics and Gynecology, Peking University People's Hospital, Beijing, China, 2 Peking University People's \\ Hospital, Beijing Key Laboratory of Female Pelvic Floor Disorders Diseases, Beijing, China
}

Background: Tumor biomechanics correlates with the progression and prognosis of endometrial carcinoma (EC). The objective of this study is to construct a risk model using the mechanical stimulus-related genes in EC.

Methods: We retrieved the transcriptome profiling and clinical data of EC from The Cancer Genome Atlas (TCGA) and Molecular Signatures Database (MSigDB). Differentially expressed mechanical stimulus-related genes were extracted from the databases, and then the least absolute shrinkage and selection operator (LASSO) regression analysis was used to construct a risk model. A nomogram integrating the genes and the clinicopathological characteristics was established and validated using the KaplanMeier survival and receiver operating characteristic $(\mathrm{ROC})$ curves to estimate the overall survival (OS) of EC patients. Protein profiling technology and immunofluorescence technique were performed to verify the connection between biomechanics and EC.

Results: In total, 79 mechanical stimulus-related genes were identified by analyzing the two databases. Based on the LASSO regression analysis, 7 genes were selected for the establishment of the risk model. This model showed a good performance in terms of the prognostic accuracy in high- and low-risk groups. The area under the ROC curves (AUC) of this model was $0.697,0.712$ and 0.723 for 3-, 5- and 7-year OS, respectively. Then, a nomogram integrating the genes of the risk model and clinical features was constructed. The nomogram could accurately predict the OS (AUC $=0.779,0.812$ and 0.806 for 3-, 5and 7-year OS, respectively). The results of the protein profiling technology and immunofluorescence revealed the expression of cytoskeleton proteins to be correlated with the Matrigel stiffness degree.

Conclusions: In summary, a risk model of 7 mechanical stimulus-related genes was identified in EC. A nomogram based on this risk model and combining the clinicopathological features to assess the overall survival of EC showed high practical value.

Keywords: endometrial cancer, mechanical stimulus, nomogram, risk model, overall survival 


\section{INTRODUCTION}

Endometrial cancer (EC) represents one of the most common gynecologic malignancies in China and around the world with an increasing incidence (1). About $75 \%$ of the EC cases are diagnosed at early stage (stage I or II), and the 5-year overall survival rate at stage III and IV in those with lymph node metastasis ranges from $20 \%$ to $71 \%$ (2). However, due to the unclear pathogenesis and molecular mechanism, the prognosis and management of EC are complicated. There is a need to explore the molecular mechanism of EC and detect the novel factors related to its diagnosis and prognosis.

Malignant cells undergo cell mechanics and particular biomechanical properties in their growth, invasion and extravasation (3). Therefore, biomechanics play a key role in the pathogenesis, diagnosis and prognosis of malignant tumors. However, current studies focus more on the cellular mechanism, while the clinical level is rarely involved.

In this study, we identified a set of EC genes that are related to the mechanical stimulus according to The Cancer Genome Atlas (TCGA) and Molecular Signatures Database (MSigDB). After comprehensive analysis of both TCGA and MSigDB databases, we found 79 mechanical stimulus-related genes from the common differentially expressed genes (DEGs) and constructed a risk model using the least absolute shrinkage and selection operator (LASSO) analysis. Afterwards, we established a nomogram combining the integrated clinicopathological features and mechanical stimulus-related risk signature to predict the overall survival (OS). We further explored the 7 key mechanical stimulus-related genes regarding their different expression and independent prognosis role. Meanwhile, the results of the protein profile immunofluorescence technique verified the interaction between the biomechanics and EC.

\section{METHODS AND MATERIALS}

\section{Data Acquisition and the Identification of Differentially Expressed Genes}

We retrieved the data of the mRNA sequences and corresponding clinicopathological characteristics of 531 uterine corpus endometrial carcinoma (UCEC) tissues and 35 normal tissues from the TCGA (https://portal.gdc.cancer.gov/) database. In addition, 208 genes associated with the response to mechanical stimulus (GO:0009612) were obtained from the Molecular Signatures Database (MSigDB http://www.gseamsigdb.org/gsea/index.jsp). We analyzed the mechanical

Abbreviations: EC, endometrial cancer; TCGA, The cancer genome atlas; MSigDB, Molecular Signatures Database; LASSO, least absolute shrinkage and selection operator; ROC, receiver operating characteristic; tdROC, timedependent receiver operating characteristic; AUC, area under the ROC curve; C-index, concordance index; UCEC, uterine corpus endometrial carcinoma; DEGs, Differentially expressed genes; HR, hazard ratio; ES, enrichment score; NES, normalized enrichment score; EEA, endometrioid endometrial adenocarcinoma; SEA, serous endometrial adenocarcinoma; FIGO, international federation of gynecology and obstetrics; G1/2/3, grade 1/2/3; LNM, lymph node metastasis; OS, overall survival. stimulus-related DEGs between EC and the normal tissues using the limma package in the $\mathrm{R}$ software, which uses the significance analysis of the microarray method, following the criteria of $\log _{2}|\mathrm{FC}|>1$ and $P$-value $<0.05$. The heatmap was plotted for the samples and DEGs were identified with the heatmap package in the $\mathrm{R}$ software. These overlapping genes were chosen for further analysis. Since the data were retrieved from open resource databases, no ethical approval was needed.

\section{Functional Enrichment Analysis of the Intersection Genes}

In this study, to investigate the underlying mechanism of the DEGs, 79 DEGs were analyzed using the following tools. The clusterProfiler Bioconductor package was used to conduct GO pathway enrichment analysis of the overlapping DEGs. Disease ontology (DO) annotates the genes based on human diseases. DO analysis was performed to reveal the clinical relevance of the obtained key genes. Then, the DOSE package of the R software was used to analyze the semantic similarity computations of the DO terms and genes, thus revealing the closeness between diseases and gene functions. A $P$-value $<0.05$ was set as the threshold value.

\section{Establishment and Verification of the Risk Model}

The LASSO regression analysis was performed to narrow the range of the DEGs and establish a predicted model using the glmnet $\mathrm{R}$ package. The LASSO regression model was used to identify the most accurate predictive genes. Next, DEGs were filtered more than 1000 times. DEGs that were discovered more than 800 times in the LASSO analysis were selected as candidate prognostic genes, and the secondary related genes were filtered out.

Then, the mechanical stimulus-related hub genes selected using the LASSO analysis were used to construct a risk signature based on the coefficient for each patient according to the following formula:

$$
R S=\sum_{i=1}^{n} \operatorname{Coef}(i) X(i)
$$

where Coef (i) denotes the coefficient, and $\mathrm{X}(\mathrm{i})$ is the $\mathrm{z}$-score transformed relative expression level for each DEG. The prognostic risk score of each patient was calculated using the formula, and according to the median score, the patients were classified into high- and low-risk groups. Then, time-dependent receiver operating characteristic (ROC) curve (tdROC) and Kaplan-Meier survival curve analyses were performed to verify this risk score system. The pheatmap $\mathrm{R}$ package was used to compare the expression of the hub genes between high- and lowrisk EC patients with different clinicopathological characteristics.

\section{Development and Validation of the Nomogram Based on the Risk Signature}

Univariate and multivariate Cox regression analyses were carried out to evaluate the independent risk factors for OS, combined with the risk score and significant clinical signature. Then, we 
constructed the nomogram using these independent risk factors by the rms R package. The nomogram calculated the prognosis risk value for each patient and classified them into low-, moderate- and high-score groups. Furthermore, calibration curves were drafted and the concordance index (C-index) was calculated to assess the accuracy of the nomogram. The KaplanMeier curves drawn using the survival and survival ROC R packages were used to further confirm the predictive specificity of the risk model, via comparing the area under the tdROC curve (AUC) in different clinicopathological subgroups (such as the age, histological type and lymph node metastasis). A $P$-value $<$ 0.05 was considered to be statistically significant.

\section{Verifying the Relationship Between Biomechanics and Endometrial Cancer Cell Line}

In order to verify the connection between the mechanical force and endometrial cancer cells, we seeded Ishikawa cells on polyacrylamide hydrogel with different stiffness degrees (500pa, $10 \mathrm{kpa}$ and $87 \mathrm{kpa}$ ). Then, we used protein profiling technology to detect the DEGs in these groups, and GO and Kyoto Encyclopedia for Genes and Genomes (KEGG) analyses were utilized to discover the enriched pathways of the DEGs. The immunofluorescence technique was conducted to observe the different expression of cytoskeleton protein (F-actin and paxillin) on hydrogel with different stiffness degrees. A $P$-value $<0.05$ was considered to be statistically significant.

\section{Cell Culture}

The EC cell line Ishikawa were obtained from laboratory stocks in Peking University People's Hospital and cultured at 5\% $\mathrm{CO}_{2}$ and $37^{\circ} \mathrm{C}$ in DMED/F12 medium (Gibco, Invitrogen, Carlsbad, CA, USA) supplied with $10 \%$ fetal bovine serum (Gibco, Invitrogen). Cell were subcultured every 2 days for cell experiments.

\section{Statistical Analysis}

Continuous variables were expressed as mean \pm SD (standard deviation) or median, while categorized variables were described by the frequency (n) and proportion (\%). Differences among variables were tested using the Student's t-test, chi-square test, one-way ANOVA tests or nonparametric tests. The log-rank test was applied to figure out the difference in the overall survival rate between the high-risk and low-risk groups. Statistical analyses were performed using the GraphPad prism 8 and R software, version 3.5.1. For all the analyses, differences were considered to be statistically significant if the $P$-values were less than 0.05 .

\section{RESULTS}

\section{Identification of Mechanical Stimulus- Related Differentially Expressed Genes and Functional Analysis}

A total of 531 endometrial cancer patients were retrieved from the TCGA database and 208 mechanical stimulus-related genes were obtained from the MSigDB, these data were analyzed as shown in the flowchart (Figure 1).

Figure 2A shows the heatmap of the expression of the DEGs in tumor tissues and normal tissues collected from the TCGA database. In the MSigDB, 208 mechanical stimulus-related genes were found in oncology tissues. There were 79 overlapping DEGs between the TCGA database and MSigDB. Among the DEGs between tumor tissues and normal tissues, 35 genes were upregulated and 44 were downregulated (Figure 2B). The volcano plot shows the mechanical stimulus-related DEGs in the datasets (Figure 2C).

GO analysis was carried out to elucidate the potential function of these 79 overlapping genes. As shown in Figure 2D, these DEGs were mostly significantly enriched in the biological process (BP) term of "response to mechanical stimulus" ( $P$-value $<0.05)$, in addition to the cellular component (CC) term of "cellular response to external stimulus" and molecular function (MF) term of "cellular response to abiotic stimulus. These results indicated that these DEGs were distributed in the mechanical stimulus-related processes and pathways, which have been proved to play a pivotal role in the tumorigenesis and progression of malignant tumors.

\section{Mechanical Stimulus-Related Risk Model Construction of the DEGs}

The LASSO regression model was conducted using the expression profiles data of 79 DEGs to establish a mechanical stimulus-related risk model for the prognosis of EC. We

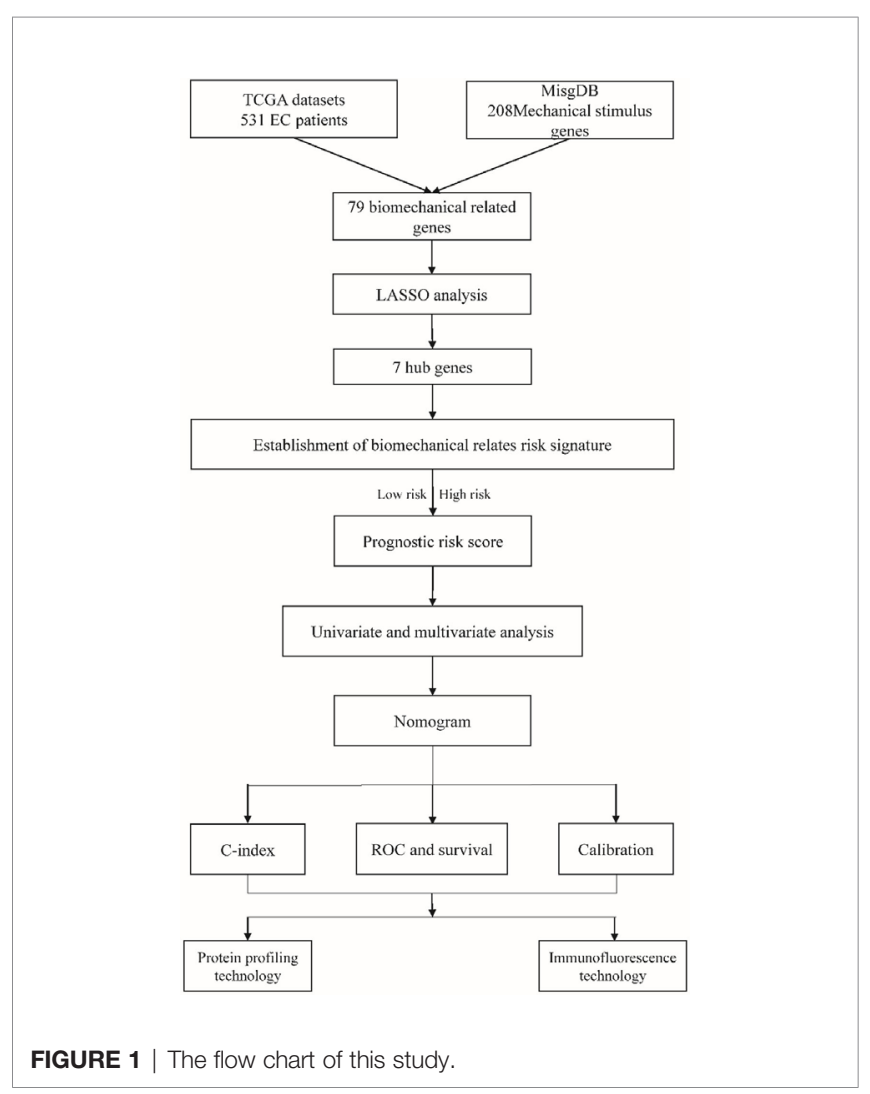




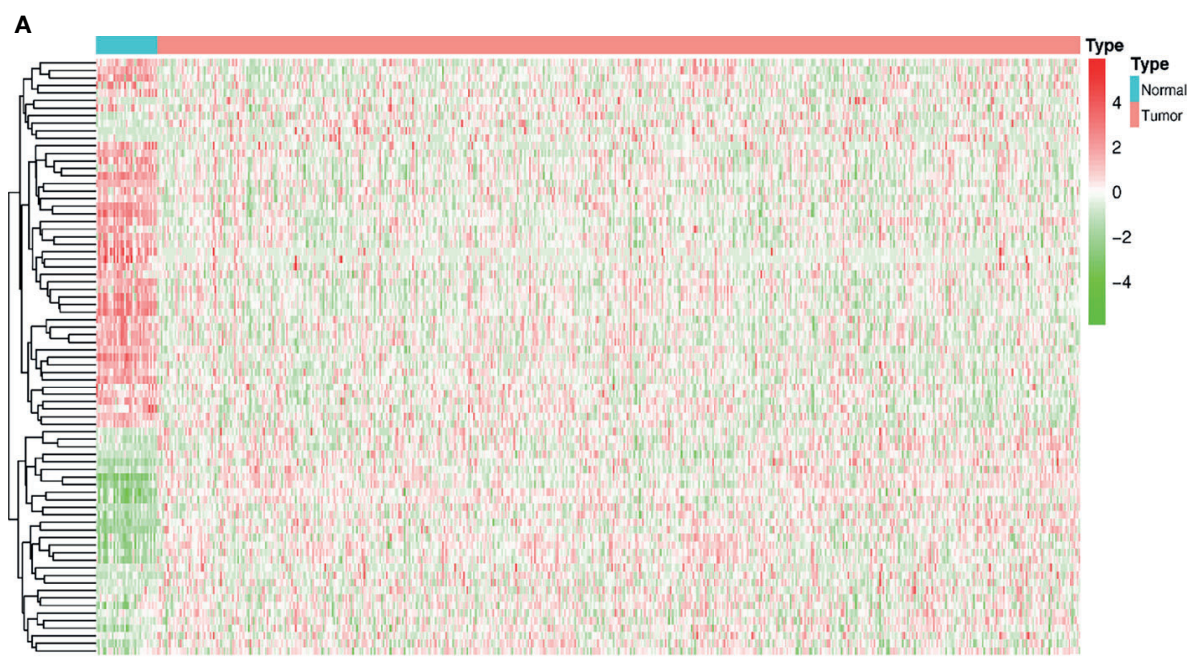

B

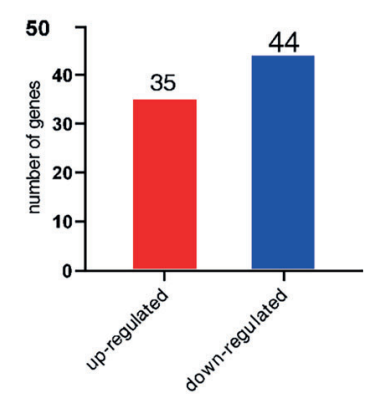

C

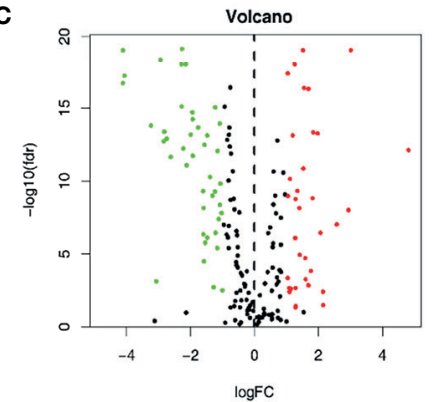

D

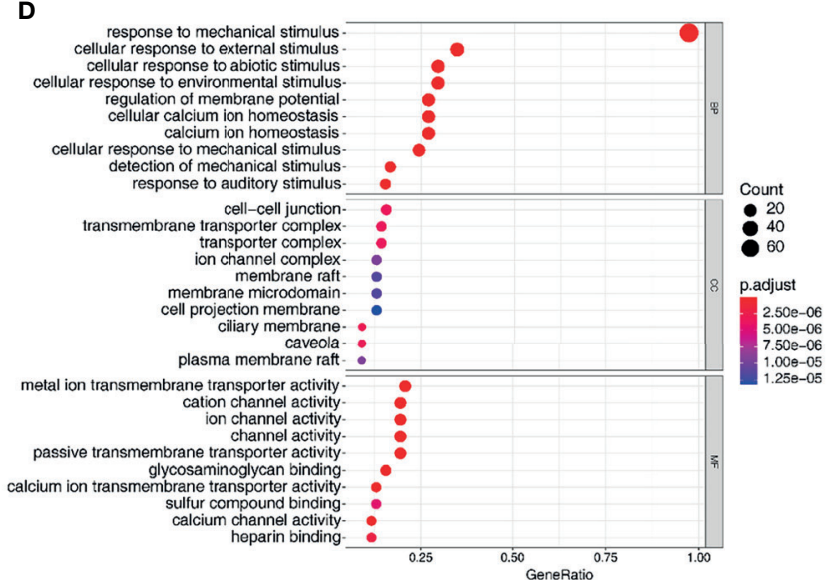

FIGURE 2 | Identification of the mechanical stimulus-related key genes. (A) Heatmap of the expression of DEGs from the TCGA database. (B) The overlapped DEGs collected from both TCGA and GO databases. (C) The volcano plot of the mechanical stimulus-related genes in the two databases. (D) GO analysis of these DEGs.

performed 6-fold cross-validation and 7 genes were finally found with regression coefficients including ASNS, DRD2, NRXN1, PTCH1, SLC2A1, SLC8A1 and USP5 (Figures 3A, B and Table 1). The expression levels of the 7 genes were significantly correlated with each other, especially between USP53 and PTCH1, USP53 and SLC2A1, NRXN1 and SLC8A1 (Figure 3C). The risk score formulation for this risk signature was established as follows: risk score $=\left(0.06826^{\star}\right.$ ASNS $)+$ $\left(0.05467^{\star} \mathrm{DRD} 2\right)+\left(0.07154^{\star} \mathrm{NRXN} 1\right)+\left(0.02918^{\star} \mathrm{PTCH} 1\right)+$ $\left(0.10020^{\star}\right.$ SLC2A 1$)+\left(0.10164^{\star}\right.$ SLC8A 1$)+\left(-0.00169^{\star}\right.$ USP53 $)$. Then, each patient was evaluated according to the risk score, and clustered into high- and low-risk groups to calculate the prognosis value of the risk score model (Figure 3D). The Kaplan-Meier survival curve showed that the survival of the low-risk group was obviously better than the high-risk group (Figure 3E). The AUC of the 7-gene risk model was 0.697 for the 3 -year survival, 0.712 for the 5 -year survival and 0.723 for the 7-year survival (Figure 3F). 
A

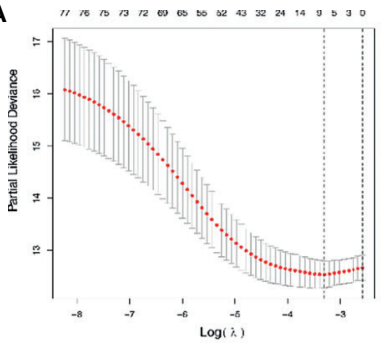

C
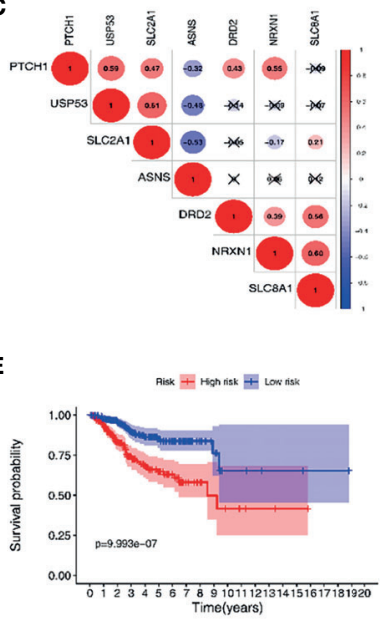

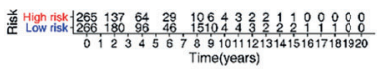

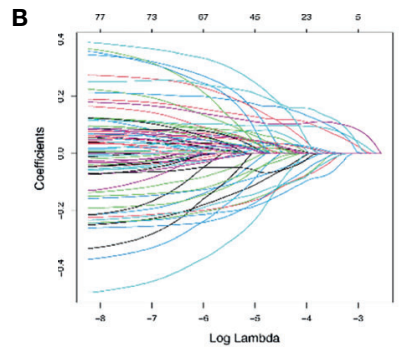

D

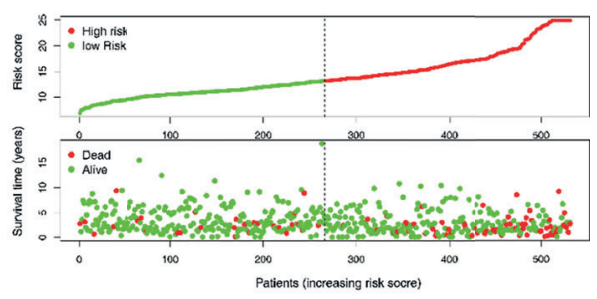

$\mathbf{F}$

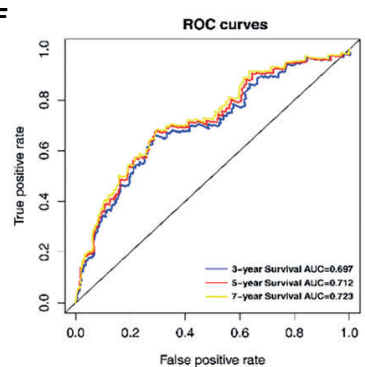

FIGURE 3 | The establishment and verification of the LASSO regression model. (A, B) the evaluation progress of gene selection in LASSO regression. (C) The interrelation of 7 key DEGs acquired from LASSO regression. (D) High- and low-risk groups according to the risk score. (E) Kaplan-Meier survival analysis of the high- and low-risk groups. (F) Time dependent ROC curves for 3-year, 5-year and 7-year survival prediction.

TABLE 1 | Mechanical stimulus-related genes and correlated coefficient value.

\begin{tabular}{lc}
\hline Gene & Coefficient \\
\hline ASNS & 0.06825528 \\
DRD2 & 0.054669638 \\
NRXN1 & 0.07153678 \\
PTCH1 & -0.029178017 \\
SLC2A1 & 0.100200637 \\
SLC8A1 & 0.10164386 \\
USP53 & -0.001690605
\end{tabular}

\section{Further Analysis of Key Genes With Clinicopathological Signatures}

The expression level of the 7 key genes in high- and low-risk patients retrieved from the TCGA database with the clinicopathological signatures were presented in the heatmap (Figure 4A and Table 2). The results revealed significant differences between the low- and high-risk groups in terms of the lymph node metastasis $(\mathrm{LNM})(P$-value $<0.001)$, cancer status $(P$-value $<0.001)$, peritoneal cytology $(P$-value $<0.01)$, recurrence $(P$-value $<0.01)$, grade $(P$-value $<0.001)$, histology $(P$-value $<0.001)$, stage $(P$-value $<0.001)$, age $(P$-value $<0.01)$ and living status $(P$-value $<0.001)$.
Furthermore, the gene set enrichment analysis (GSEA) was conducted to investigate the role of these key genes in the endometrial cancer tumorigenesis mechanism. As shown in Figures $4 \mathbf{B}-\mathbf{D}$, these genes might play a crucial role in the regulation of actin cytoskeleton $(\mathrm{ES}=0.402, \mathrm{NOM} P$-value $=$ $0.004)$, tight junction $(\mathrm{ES}=0.411, \mathrm{NOM} P$-value $=0.004)$ and focal adhesion $(\mathrm{ES}=0.448$, NOM $P$-value $=0.007)$; all of these cell signal pathways were related to the mechanical stimulus. After all, the risk group built on the mechanical stimulus-related signature of 7 key genes might be distinguishable when the EC patients were classified according to our high- and low-risk models, and the mechanism might be involved in the pathway responding to mechanical stimulus.

\section{Establishment and Evaluation of a Prognostic Nomogram}

Univariate and multivariate Cox regression analyses were used to distinguish the independent risk factors by developing a nomogram used to further predict the clinical outcome of EC patients with the mechanical stimulus-related risk model. Figure 5A showed the evaluation of the univariate analysis, and Figure 5B showed the result of multivariate analysis, suggesting that the age (Hazard ratio(HR) $=1.023,95 \%$ 


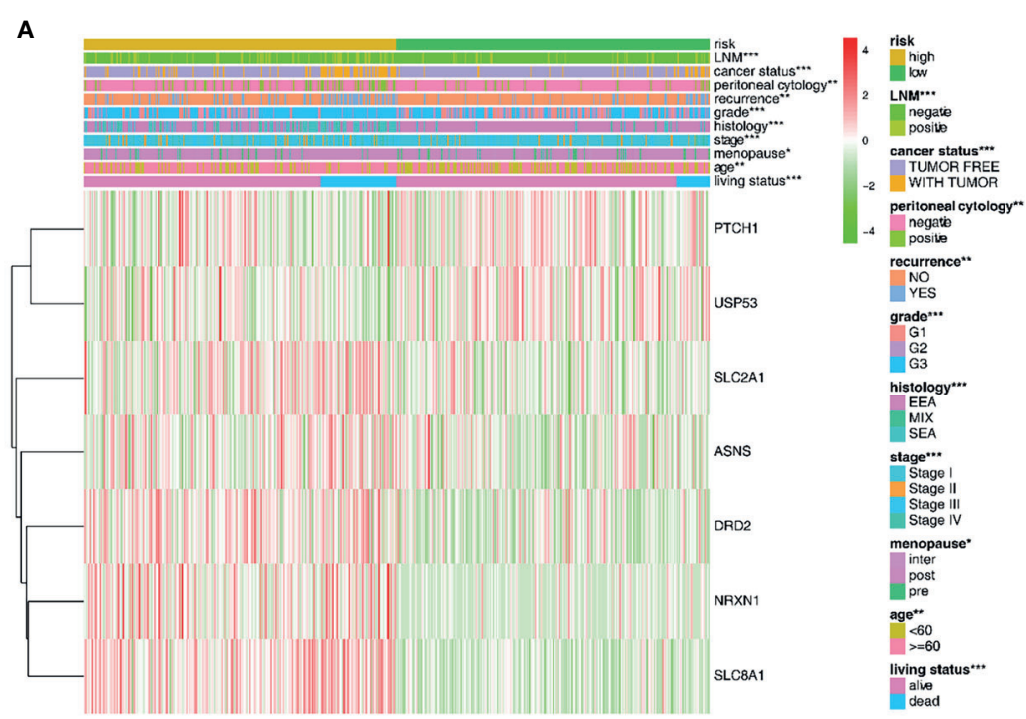

B

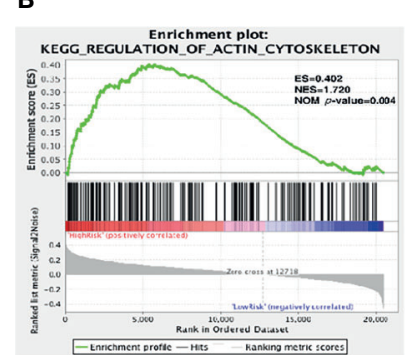

C

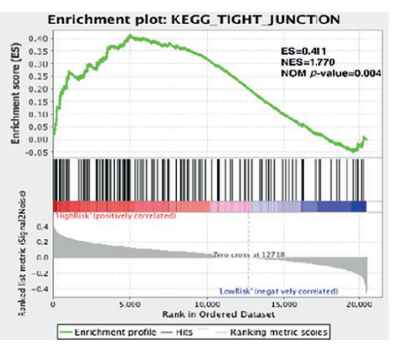

D

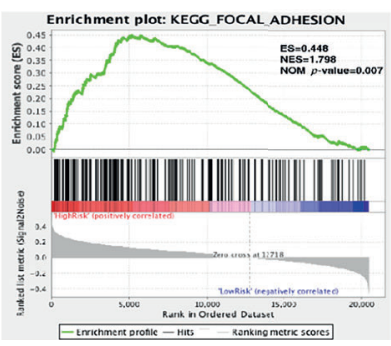

FIGURE 4 | Predictive accuracy in high- and low-risk groups with differential clinicopathological signatures. (A) Heatmap with clinicopathological features in the two groups. Chi-square test was used for the correlation between clinical significance and the cluster. ${ }^{*} p<0.05,{ }^{* *} p<0.01$ and ${ }^{* * *} p<0.001$. (B-D) GSEA analysis showing that the higher expression genes in the high-risk group are enriched in the regulation of actin cytoskeleton, tight junction and focal adhesion.

Confidence Interval $(\mathrm{CI}): 1.002-1.045, P$-value $=0.033)$, stage $(\mathrm{HR}=1.368,95 \% \mathrm{CI}: 1.053-1.777, P$-value $=0.019)$, grade $(\mathrm{HR}=$ 1.883 95\%CI: $1.206-2.942, P$-value $=0.005)$, peritoneal cytology $(\mathrm{HR}=1.970,95 \% \mathrm{CI}: 1.091-3.556, P$-value $=0.024)$ and risk signature $(\mathrm{HR}=1.941,95 \% \mathrm{CI}: 1.140-3.305, P$-value $=0.015)$ were found to be independent risk factors for the overall survival of EC patients. Then, we designed a nomogram to predict the probability of OS (Figure 5C). In the nomogram, each predictor was assigned a score, based on the multivariate analysis results, and a total of 5 factors were integrated in the nomogram to predict the OS of EC patients. The calibration curve suggested that the nomogram that predicted the survival rate was close to the actual situation for all of the 3-, 5-, and 7-year survival (Figure 5D).

In addition, we categorized the patients into 3 subgroups according to their total points and further tested the survival assessment model using the Kaplan-Meier analysis in both the whole cohort and subgroups. The results showed that this nomogram could perform well in partitioning the patients in the whole groups (Figure 5E). The AUC of the nomogram was $0.779,0.812$ and 0.806 for the 3-, 5- and 7-year OS, respectively (Figure 5F). These findings revealed that this risk signature- based nomogram had definite reliability and specificity in evaluating the prognosis.

\section{Further Verification of the Nomogram in Different Clinicopathological Subgroups}

To further evaluate and verify the survival assessment model, we conducted the Kaplan-Meier survival analysis in the following different clinicopathological subgroups of the patients: age subgroups, age $<60$ and age $\geq 60$; histological type subgroups, EEA and other types; lymph node metastasis subgroups, negative and positive; peritoneal cytology subgroups, negative and positive. The tests showed that the predictive capability of the survival assessment model was effective in all the clinicopathological subgroups of EC patients (Figures 6A-H). Thus, this mechanical stimulus-related risk model had a certain reliability and practicability in evaluating the prognosis.

\section{Excavation of the Relationship Between Biomechanical Genes and the Pathogenesis of EC}

In order to verify the prediction results, we conducted experiments to explore the effects of different mechanical stimulations on the 
TABLE 2 | Clinicopathological signatures of patients in high- and low-risk.

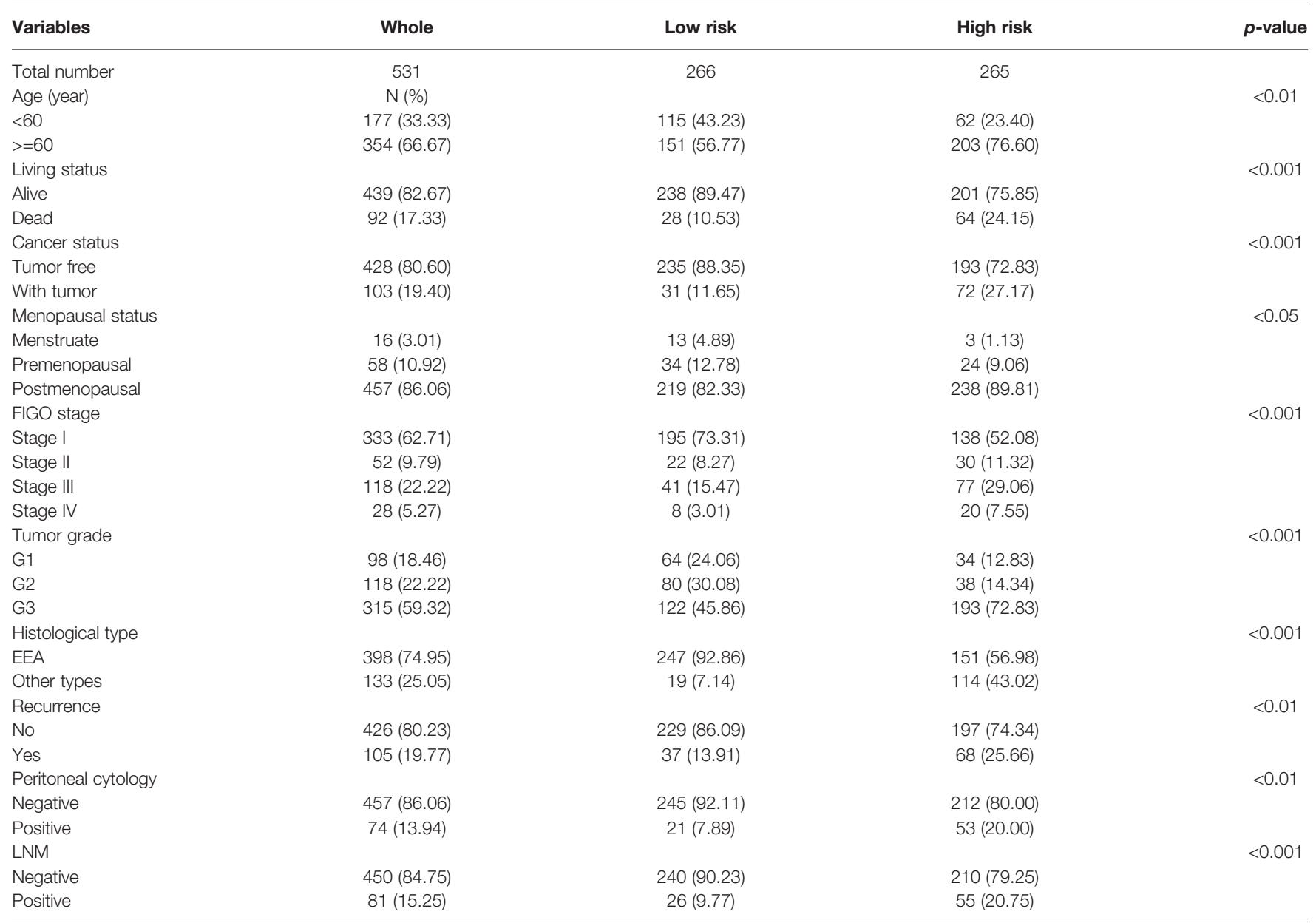

EEA, endometrioid endometrial adenocarcinoma; FIGO, international federation of gynecology and obstetrics; LNM, lymph node metastasis.

function of EC cells. The results of the protein profiling technology in different stiffness degrees $(500 \mathrm{kPa}, 10 \mathrm{kPa}$ and $87 \mathrm{kpa})$ indicated a clearly different expression level of the proteins in these groups (Figure 7A).

Moreover, GO analysis demonstrated that the function of these proteins obviously concentrated on the regulation of actin cytoskeleton (Figure 7B) and the enriched bp term was the "cytoskeletondependent intracellular transport", cc term was the "mitochondrial inner membrane" and $\mathrm{mf}$ term was the "cell adhesion molecule binding" (Figure 7C). Furthermore, the Ishikawa cells seeded in the three groups showed a significant difference in the cytoskeleton proteins (F-actin and Paxillin), with the increase in stiffness, the expression of cytoskeleton protein was strengthened. Meanwhile, the number of pseudopodia growing with the increase in stiffness indicated the enhancement of cell motility (Figure 8). These results demonstrated that biomechanical genes might play a crucial role in the malignant process of EC, especially in metastasis and invasion.

\section{DISCUSSION}

Endometrial cancer is one of the most common gynecologic malignant tumors with an increasing incidence of high-grade, clinically aggressive tumors (4). At the advanced stage, the rate of 5 -year overall survival approximately reaches 15\% (5). Therefore, it is of great significance to discover the diagnosis and prognosis biomarkers and to seek out the mechanism behind the metastasis and invasion of EC. Nowadays, bioinformatics analysis is widely used to explore the crucial genes in the pathogenesis and prognosis of various types of tumors. Besides, the nomogram model is being more used to predict the prognosis of cancer using the clinicopathological signatures. A previous study (6) used bioinformatics analysis and discovered that the FOXL2, TCF4 and NR2F2 transcription factors could serve as biomarkers in the prognosis and potential targets in clinical therapy. Yet, biomarkers explored by most of the previous studies focused on the molecular factors. In our study, clinical and molecular data combined with the genomic characteristics were explored to develop the prognostic model and improve the accuracy.

In the progress of cancer growth, metastasis, invasion and adhesion, malignant cells demand particular mechanical properties (6). Tumor biomechanics may regulate the cytoskeleton signaling pathway to induct the malignant behavior. A previous study expounded that Talin2 may bind to $\beta$-integrin to regulate the tumor cell migration and invasion, 
A

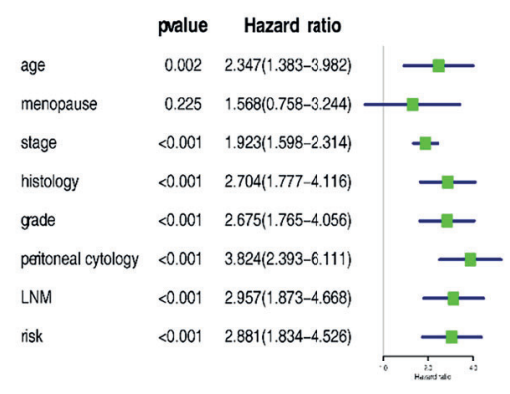

C

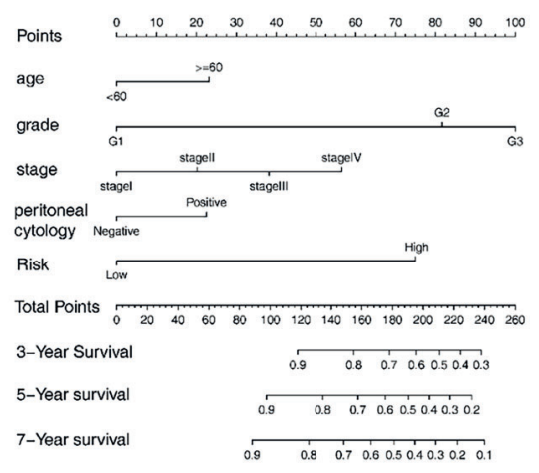

E

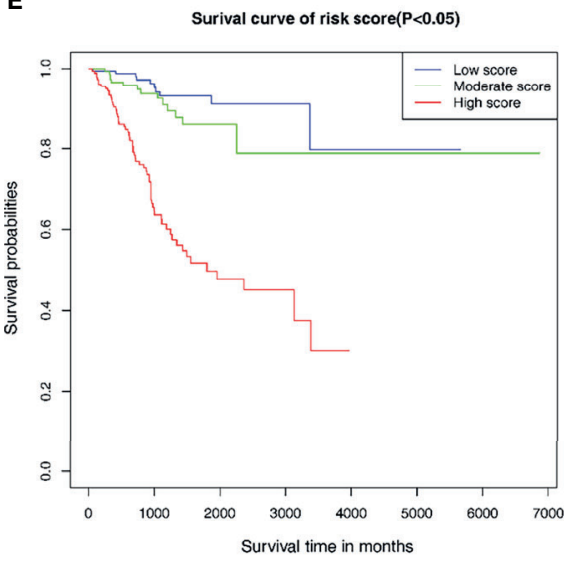

B

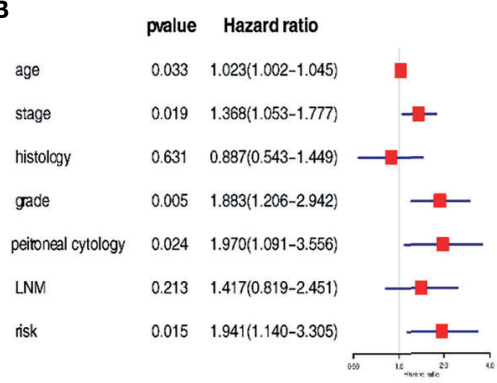

D

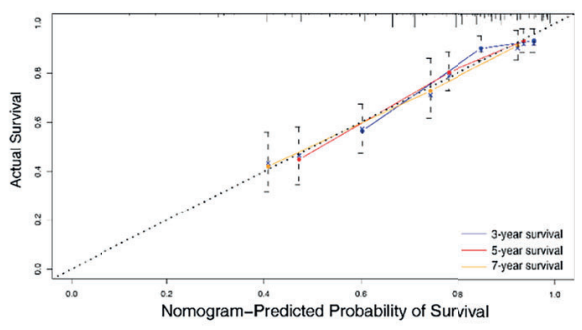

$\mathbf{F}$

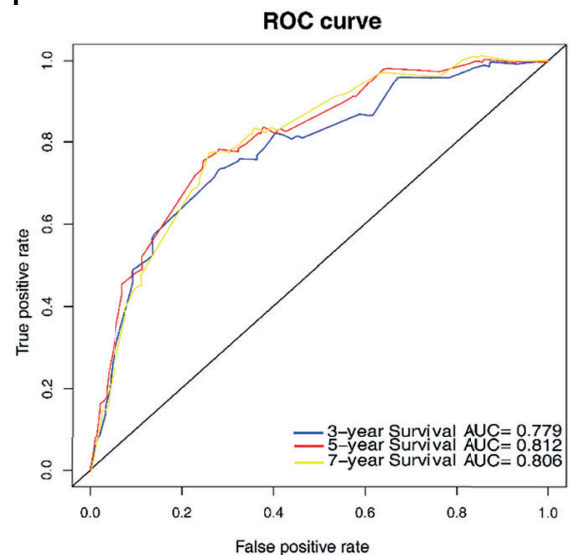

FIGURE 5 | Establishment of the nomogram for prognostic prediction. (A) Univariate analyses of the risk model with clinicopathological features. (B) Multivariate analysis of the risk model with clinicopathological features. (C) Nomogram including the 7 key genes, risk score and clinicopathological signatures. (D) Calibration plot of the nomogram prediction possibility. (E) Kaplan-Meier survival analysis of the patients in the risk score. (F) Time-dependent ROC curve in the risk model.

playing a key role in cancer cell invasion (7). In breast cancer, researchers also found that the remodeling and reorganization of cytoskeleton and cell-matrix could alter the traction forces, thereby modulating the cell motility behavior (8). However, the relationship between biomechanics and EC has rarely been investigated. In this study, we found that the mechanical stimulus-related genes could act as predictive factors in the prognosis of EC. Then, we verified their biological functions in the development of cancer. Our results indicated that these key genes were related to the cytoskeleton regulation.
The TCGA database was used to retrieve the genomic data of endometrial cancer patients, and 79 overlapped genes were found. Using the LASSO analysis, 7 key genes (ASNS, DRD2, NRXN1, PTCH1, SLC2A1, SLC8A1 and USP5) were sought out to establish the prognosis model. ASNS is involved in the synthesis of asparagine, progression of the cell cycle and sensitivity to cisplatin of malignant tumors (9). DRD2 encodes a kind of dopamine receptors and represents a tumor suppressor educating $\mathrm{M} 1$ macrophages by the NF- $\mathrm{\kappa B}$ signaling pathway (10). NRXN1 encodes a membrane protein that is interrelated 

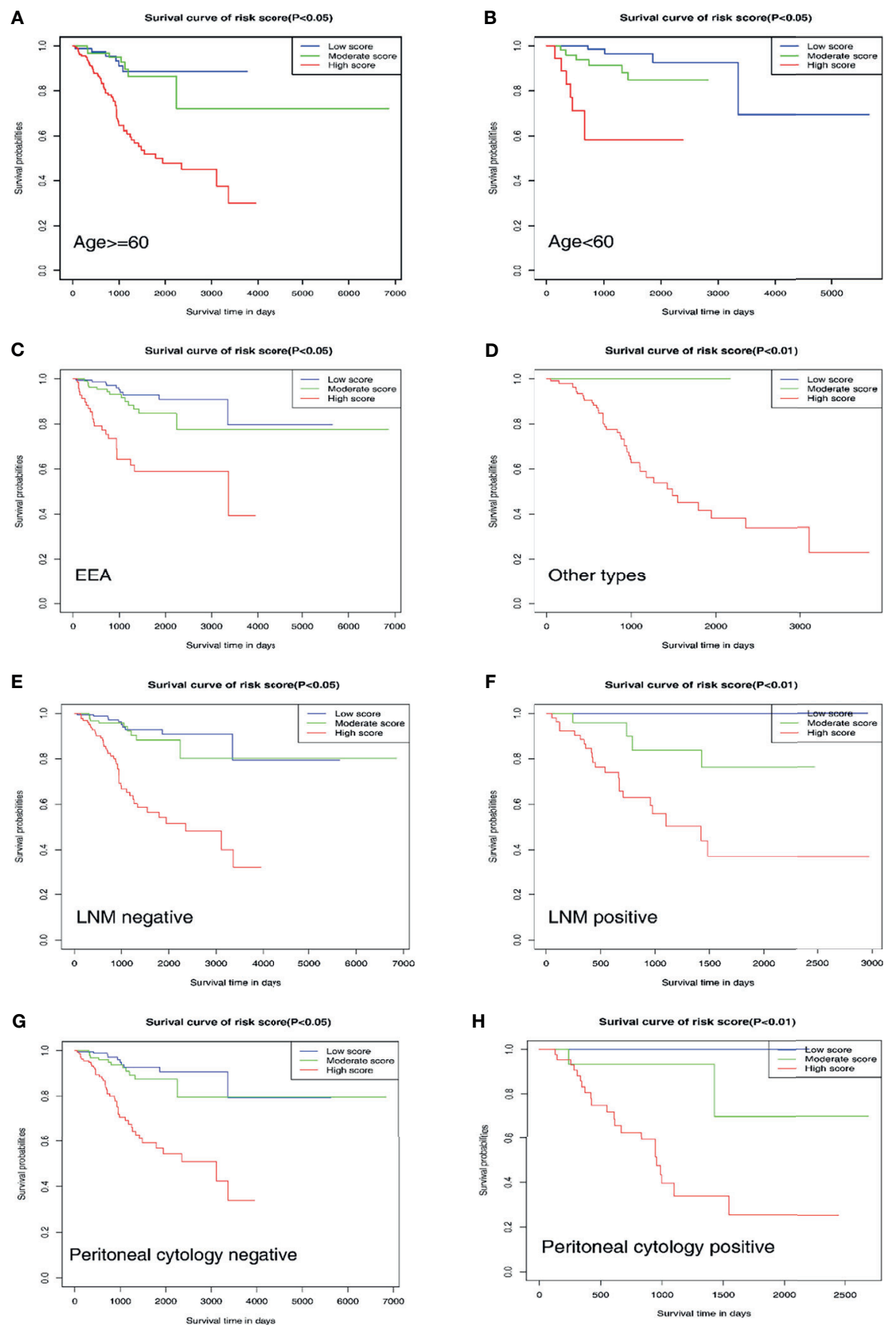

FIGURE 6 | Prognostic value of the nomogram in different clinicopathological subgroups. (A, B) Age subgroups. (C, D) Histological type subgroups. (E, F) Lymph node metastasis subgroups. (G, H) Peritoneal cytology subgroups.

with the transfer of $\mathrm{Ca}^{2+}$ (11). Previous studies demonstrated that PTCH1 could induct the malignant cell stemness and facilitate the cell autophagy $(12,13)$. SLC2A1 and SLC8A1 both belong to the solute carrier family. SLC2A1 encodes the glucose transporter, which is associated with the abnormal metabolism during cancer cell invasion (14), while SLC8A1 participates in the migration of tumor cells via regulating $\mathrm{Na}^{+} / \mathrm{Ca}^{2+}$ exchange (15), it is also involved in the endometrial tissue cellular heterogeneity (16). Finally, USP5 encodes a metabolism protein that is highly expressed in many kinds of cancer, such as breast, prostate and hepatic cancer (17). The results of our study show that the functions of these genes are enriched in the biomechanical signal pathways, including the regulation of actin cytoskeleton, tight junction and focal adhesion. 

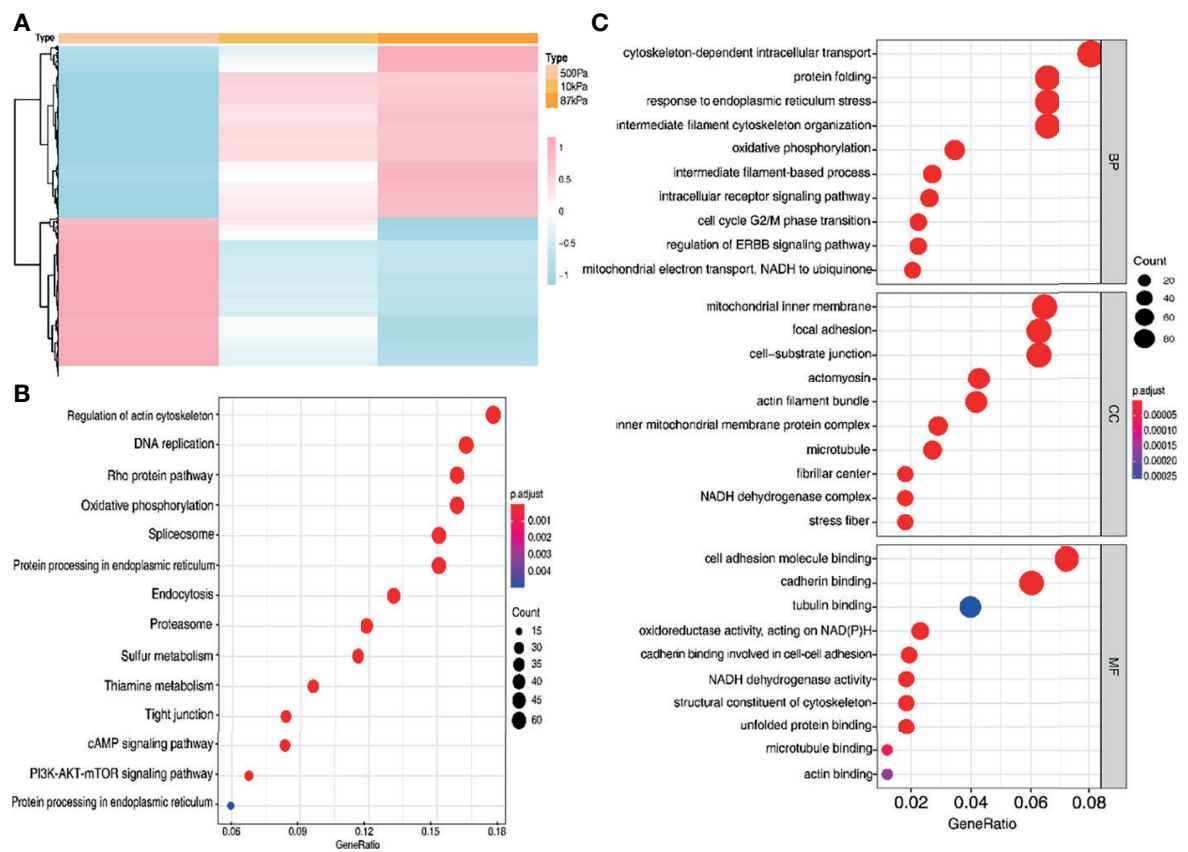

FIGURE 7 | The protein expression of the Ishikawa cells on polyacrylamide hydrogel with different stiffness degrees. (A) Heatmap of the proteins with different expression levels in hydrogel. (B, C) GO analysis of the proteins with different expression levels.

A

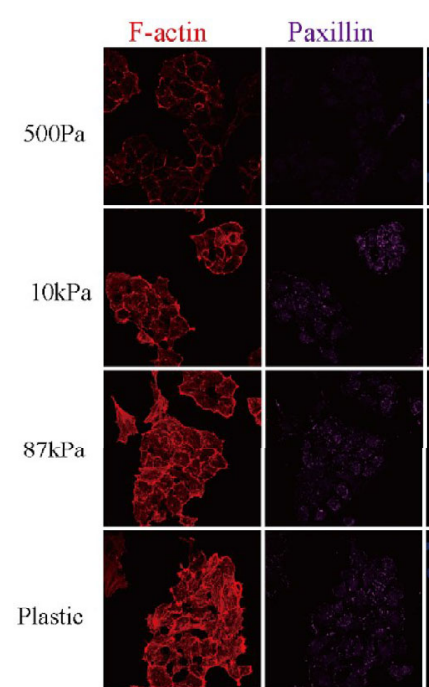

ishikawa

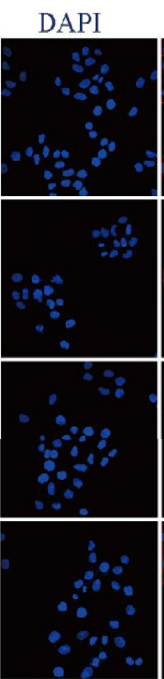

Merge

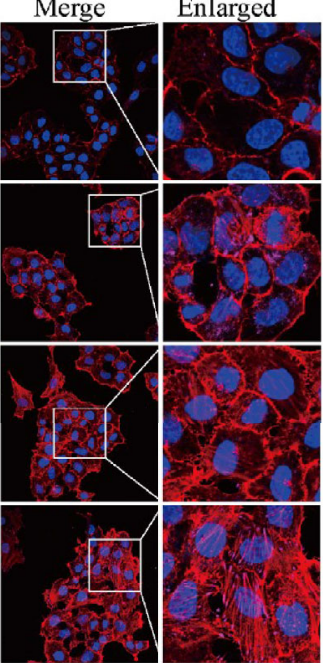

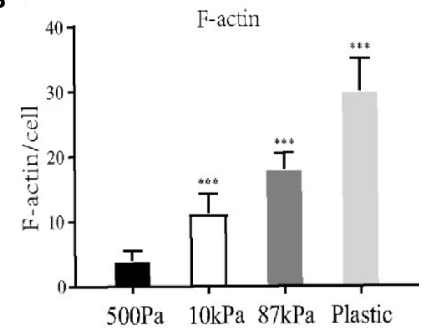

C

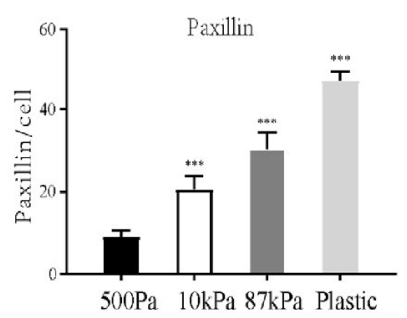

FIGURE 8 | Expression of the Ishikawa cytoskeleton proteins on Matrigel with different stiffness degrees. (A) The imagines of F-actin and paxillin. (B, C ) The expression of F-actin, paxillin and pseudopodia in Ishikawa cells. ${ }^{\star \star} p<0.01$ and ${ }^{\star \star *} p<0.001$.

In addition, our study analyzed much data from the TCGA database and focused on the gene expression of mechanical stimulusrelated genes. Compared with previous studies on EC prediction biomarkers, in our study, a nomogram was used as a graphical tool to evaluate the prediction factors more tangibly and intuitively interpret the predictors with the clinical signatures (18). Our nomogram displayed that the biomechanical genes could be independent prognostic predictors of EC, which was verified via the Kaplan-Meier analysis in the clinicopathological groups, especially in the age, histological type, lymph node metastasis and peritoneal cytology subgroups. 
This study showed that mechanical stimulus played an important role in endometrial cancer prognosis. Moreover, the tumor cells were subjected to a variety of mechanical forces, and the stiffness of the extracellular matrix is one of the main forces (19). In order to further verify the relationship between mechanical factors and endometrial cancer, we used polyacrylamide hydrogels with different stiffness degrees to simulate the extracellular matrix with different stiffness, then the protein profiling technology analyzed the protein expression of Ishikawa cells seeded in polyacrylamide hydrogels with different stiffness degrees. Through GO analysis, we found these DEGs to be enriched in the pathway of the regulation of actin cytoskeleton. Cytoskeleton is crucial for cell deformation and shape changing, internal and external forces could affect the cell mechanical characteristics via regulating cytoskeleton (20). Besides, cytoskeleton is the inductor and presenter of cell migration and invasion in malignant tumors (21). Our group previously reported that transient receptor potential vanilloid 4 (TRPV4) was involved in EC cells migration through cytoskeletal regulation (22). We further demonstrated the expression of cytoskeleton with different stiffness degrees of hydrogels by cellular experiments, results showed that with the increase in stiffness, the expression of cytoskeleton protein was enhanced and the number of pseudopodia was increased. This result illustrated that the increasing stiffness of hydrogels could promote the motility of EC cells by regulating cytoskeleton.

Taken together, our study identified 7 mechanical stimulusrelated genes via the LASSO analysis and set up a nomogram predictive model for EC patients. In the validation group, the OS of the high-risk group associated with the age, lymph node metastasis, ascites metastasis and FIGO stage were obviously worse than the patients in the low-risk group. Our study showed that cellular mechanics represented a considerable factor participating in the progression and invasion of EC.

\section{CONCLUSION}

In summary, we constructed a prognostic risk model with 7 mechanical stimulus-related genes and established a prognostic

\section{REFERENCES}

1. Siegel RL, Miller KD, Fuchs HE, Jemal A. Cancer Statistics, 2021. CA Cancer J Clin (2021) 71(1):7-33. doi: 10.3322/caac.21654

2. Morice P, Leary A, Creutzberg C, Abu-Rustum N, Darai E. Endometrial Cancer. Lancet (2016) 387(10023):1094-108. doi: 10.1016/S0140-6736(15)00130-0

3. Gensbittel V, Kräter M, Harlepp S, Busnelli I, Guck J, Goetz JG. Mechanical Adaptability of Tumor Cells in Metastasis. Dev Cell (2021) 56(2):164-79. doi: 10.1016/j.devcel.2020.10.011

4. Lu KH, Broaddus RR. Endometrial Cancer. N Engl J Med (2020) 383 (21):2053-64. doi: 10.1056/NEJMra1514010

5. Lee YC, Lheureux S, Oza AM. Treatment Strategies for Endometrial Cancer: Current Practice and Perspective. Curr Opin Obstetrics Gynecol (2017) 29 (1):47-58. doi: 10.1097/GCO.0000000000000338

6. Gensbittel V, Busnelli I, Osmani N, Goetz JG. Nanoluminal Signaling Shapes Collective Metastasis. Trends Cancer (2021) 7(1):9-11. doi: 10.1016/ j.trecan.2020.11.003 prediction tool in the form of an integrative nomogram that could accurately and specifically forecast the clinical outcome of EC. We also explored the functions EC cells on hydrogels with different stiffness degrees. Although the clear mechanism of biomechanics regulating the progress of EC remains in need for further exploration, this study contributes to promoting the research on the mechanisms of tumor malignant behavior in EC.

\section{DATA AVAILABILITY STATEMENT}

The raw data supporting the conclusions of this article will be made available by the authors, without undue reservation.

\section{AUTHOR CONTRIBUTIONS}

Writing-Original Draft Preparation, XX. Data Curation, XL. Writing-Review and Editing, JZ and JW. All authors contributed to the article and approved the submitted version.

\section{FUNDING}

This study is supported by the National Key Technology R\&D Program of China (Grant Number. 2019YFC1005200 and 2019YFC1005201), Peking University Medicine Fund of Fostering Young Scholars' Scientific \& Technological Innovation (Grant Number. BMU2021PYB012 and BMU2021MX006) and National Natural Science Foundation of China (Grant Number. 81874108 and 82072861).

\section{ACKNOWLEDGMENTS}

The authors would like to express their gratitude to EditSprings (https://www.editsprings.com/) for the expert linguistic services provided.

7. Qi L, Jafari N, Li X, Chen Z, Li L, Hytönen VP, et al. Talin2-Mediated Traction Force Drives Matrix Degradation and Cell Invasion. J Cell Sci (2016) 129(19):3661-74. doi: 10.1242/jcs.185959

8. Fokkelman M, Balcıoglu HE, Klip JE, Yan K, Verbeek FJ, Danen EH, et al. Cellular Adhesome Screen Identifies Critical Modulators of Focal Adhesion Dynamics, Cellular Traction Forces and Cell Migration Behaviour. Sci Rep (2016) 6:31707. doi: 10.1038/srep31707

9. Hemmingsson O, Kao G, Still M, Naredi P. ASNA-1 Activity Modulates Sensitivity to Cisplatin. Cancer Res (2010) 70(24):10321-8. doi: 10.1158/00085472.CAN-10-1548

10. Tan Y, Sun R, Liu L, Yang D, Xiang Q, Li L, et al. Tumor Suppressor DRD2 Facilitates M1 Macrophages and Restricts NF- $\kappa b$ Signaling to Trigger Pyroptosis in Breast Cancer. Theranostics (2021) 11(11):5214-31. doi: $10.7150 /$ thno. 58322

11. Cosemans N, Vandenhove L, Vogels A, Devriendt K, Van Esch H, Van Buggenhout G, et al. The Clinical Relevance of Intragenic NRXN1 Deletions. J Med Genet (2020) 57(5):347-55. doi: 10.1136/jmedgenet-2019-106448 
12. Zhu R, Gires O, Zhu L, Liu J, Li J, Yang H, et al. TSPAN8 Promotes Cancer Cell Stemness via Activation of Sonic Hedgehog Signaling. Nat Commun (2019) 10(1):2863. doi: 10.1038/s41467-019-10739-3

13. Tang S, Wang Y, Xie G, Li W, Chen Y, Liang J, et al. Regulation of Ptch1 by miR-342-5p and FoxO3 Induced Autophagy Involved in Renal Fibrosis. Front Bioeng Biotechnol (2020) 8:583318. doi: 10.3389/fbioe.2020.583318

14. Shang R, Wang M, Dai B, Du J, Wang J, Liu Z, et al. Long Noncoding RNA SLC2A1-AS1 Regulates Aerobic Glycolysis and Progression in Hepatocellular Carcinoma via Inhibiting the STAT3/FOXM1/GLUT1 Pathway. Mol Oncol (2020) 14(6):1381-96. doi: 10.1002/1878-0261.12666

15. Huang T, Xu T, Wang Y, Zhou Y, Yu D, Wang Z, et al. Cannabidiol Inhibits Human Glioma by Induction of Lethal Mitophagy Through Activating TRPV4. Autophagy (2021) 17:1-15. doi: 10.1080/15548627. 2021.1885203

16. Suhorutshenko M, Kukushkina V, Velthut-Meikas A, Altmäe S, Peters M, Mägi R, et al. Endometrial Receptivity Revisited: Endometrial Transcriptome Adjusted for Tissue Cellular Heterogeneity. Hum Reprod (Oxford England) (2018) 33(11):2074-86. doi: 10.1093/humrep/dey301

17. Ning F, Xin H, Liu J, Lv C, Xu X, Wang M, et al. Structure and Function of USP5: Insight Into Physiological and Pathophysiological Roles. Pharmacol Res (2020) 157:104557. doi: 10.1016/j.phrs.2019.104557

18. Park SY. Nomogram: An Analogue Tool to Deliver Digital Knowledge. J Thorac Cardiovasc Surg (2018) 155(4):1793. doi: 10.1016/j.jtcvs.2017.12.107

19. Eble JA, Niland S. The Extracellular Matrix in Tumor Progression and Metastasis. Clin Exp Metastasis (2019) 36(3):171-98. doi: 10.1007/s10585019-09966-1
20. Fletcher DA, Mullins RD. Cell Mechanics and the Cytoskeleton. Nature (2010) 463(7280):485-92. doi: 10.1038/nature08908

21. Price LS, Collard JG. Regulation of the Cytoskeleton by Rho-Family GTPases: Implications for Tumour Cell Invasion. Semin Cancer Biol (2001) 11(2):16773. doi: $10.1006 /$ scbi.2000.0367

22. Li X, Cheng Y, Wang Z, Zhou J, Jia Y, He X, et al. Calcium and TRPV4 Promote Metastasis by Regulating Cytoskeleton Through the RhoA/ROCK1 Pathway in Endometrial Cancer. Cell Death Dis (2020) 11(11):1009. doi: 10.1038/s41419-020-03181-7

Conflict of Interest: The authors declare that the research was conducted in the absence of any commercial or financial relationships that could be construed as a potential conflict of interest.

Publisher's Note: All claims expressed in this article are solely those of the authors and do not necessarily represent those of their affiliated organizations, or those of the publisher, the editors and the reviewers. Any product that may be evaluated in this article, or claim that may be made by its manufacturer, is not guaranteed or endorsed by the publisher.

Copyright (c) $2021 \mathrm{Xu}, \mathrm{Li}$, Zhou and Wang. This is an open-access article distributed under the terms of the Creative Commons Attribution License (CC BY). The use, distribution or reproduction in other forums is permitted, provided the original author(s) and the copyright owner(s) are credited and that the original publication in this journal is cited, in accordance with accepted academic practice. No use, distribution or reproduction is permitted which does not comply with these terms. 